\title{
A Case of a Pigmented Epithelioid Melanocytoma on a Mucosal Site
}

\author{
Alice Ramondetta ${ }^{1}$, Simone Ribero $^{1}$, Luca Conti ${ }^{1}$, Pietro Quaglino ${ }^{1}$, Paolo Broganelli ${ }^{1}$
}

1 Dermatology Clinic and Surgical Pathology Section, Department of Medical Sciences, University of Turin, Italy

Key words: melanocytoma, epithelioid cells, vulva, genital dermatopathology, melanocytic lesion

Citation: Ramondetta A, Ribero S, Conti L, Quaglino P, Broganelli P. A case of a pigmented epithelioid melanocytoma on a mucosal site. Dermatol Pract Concept. 2020;10(4):e2020070. DOI: https://doi.org/10.5826/dpc.1004a70

Accepted: April 21, 2020; Published: October 26, 2020

Copyright: (2020 Ramondetta et al. This is an open-access article distributed under the terms of the Creative Commons Attribution License BY-NC-4.0, which permits unrestricted noncommercial use, distribution, and reproduction in any medium, provided the original author and source are credited.

Funding: None.

Competing interests: The authors have no conflicts of interest to disclose.

Authorship: All authors have contributed significantly to this publication.

Corresponding author: Simone Ribero, MD, PhD, Dermatology Clinic, Department of Medical Sciences, University of Turin, via Cherasco 23, 10126, Italy. Email: simone.ribero@unito.it

\section{Introduction}

Pigmented epithelioid melanocytoma (PEM) is an uncommon and recently described entity with unknown biologic behavior. It is a melanocytic tumor showing overlapping features of both an atypical epithelioid blue nevus and a lowgrade "animal-type melanoma."

PEM occurs over a broad age range, with a predilection for children and young adults, and clinically appears as a macular, popular, or nodular lesion. Dermoscopically, PEM appears as a polymorphic lesion, characterized by homogeneous blue pigmentation and a combination of black, brown, and white colors. Histopathologically, it is characterized by a dermal proliferation of heavily pigmented, both dendritic and spindle/epithelioid melanocytes, admixed with slightly larger, plumper, and less pigmented epithelioid cells [1]. Involvement of the regional nodes has been reported but usually with no further spread of the disease. No histological criteria are predictive of metastatic behavior.

\section{Case Presentation}

We describe a case of vulvar mucosal PEM, that occurred on the right small lip of a 50-year-old woman. Clinically it appeared as an intensely pigmented, brown-black papule about $1 \mathrm{~cm}$ in size. On dermoscopy, we observed many irregular, black blotches surrounded by a whitish blue veil. No atypical vascular feature was detected. Due to its globular structure and black-bluish color and considering the age of the patient and rapid onset, the lesion resembled a Spitz nevus or an atypical Spitz tumor (Figure 1, A and B). However, spitzoid lesions do not show these dermoscopic features. Therefore, an excisional biopsy was performed.

The histological examination showed an acanthotic epidermis with a slightly warty profile overlying a large dermal nodular lesion with blurred borders consisting of hyperpigmented epithelioid and spindle dendritic melanocytes arranged in perivascular and periadnexal bundles, mixed with epithelioid nevoid cells and numerous melanophages. No significant atypia, mitosis, or necrosis was appreciated. 


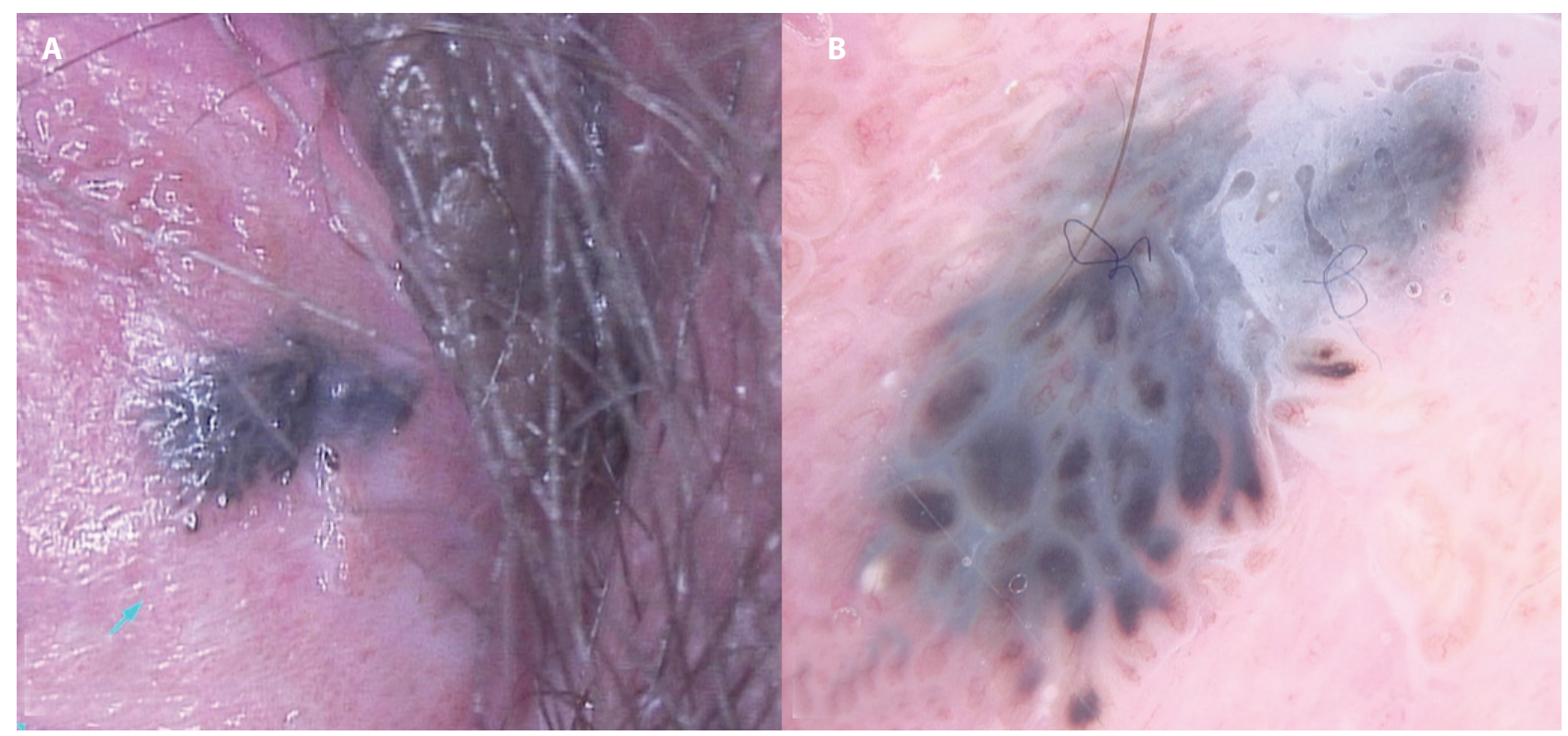

Figure 1. (A) Clinical appearance: oval papule, brown-black in color, intensely pigmented, about $1 \mathrm{~cm}$ in size. (B) Dermoscopy: many irregular, black blotches surrounded by a whitish blue veil. No atypical vascular feature was detected.

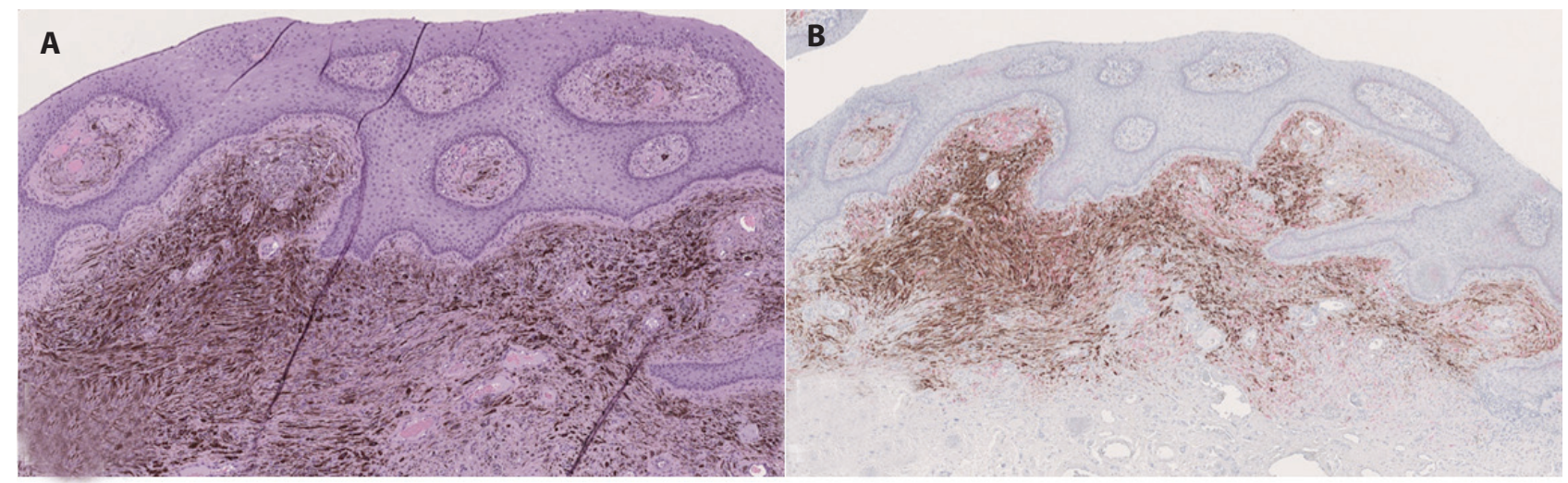

Figure 2. (A) Histopathological specimen: acanthotic epidermis with a slightly warty profile overlying a large dermal nodular lesion with blurred limits consisting of hyperpigmented epithelioid and spindle dendritic melanocytes arranged in perivascular and periadnexal bundles, mixed with epithelioid nevoid cells and numerous melanophages. No significant atypia, mitosis, or necrosis was appreciated. Immunohistochemical staining: HMB-45, Melan-A (B) and p16 (all expressed), KI-67 (1\%).

Immunohistochemical staining was performed. While HMB45 and Melan-A were positive, p16 was expressed on the entire lesion, and KI-67 showed low activity (1\%) (Figure 2, $\mathrm{A}$ and $\mathrm{B})$.

\section{Conclusions}

Pigmented lesions of mucosal sites usually can be difficult to interpret. PEM is a rare entity, and we report on a case of a female genital mucosal variant. Uehara et al published a case of PEM on the glans [2]. PEM could mimic both benign and malignant lesions, from which it must be distinguished, albeit with difficulty.

\section{References}

1. Moscarella E, Ricci R, Argenziano G, et al. Pigmented epithelioid melanocytoma: clinical, dermoscopic and histopathological features. Br J Dermatol. 2016;174(5):1115-1117. https://doi. org/10.1111/bjd.14322. PMID:26614732.

2. Uehara M, Sato S, Kato A, et al. A rare case of pigmented epithelioid melanocytoma on the penis as a divided nevus. Eur J Dermatol. 2014;24(2):248-249. https://doi.org/10.1684/ ejd.2014.2272. PMID:24723648. 